\title{
A Case of Steatocystoma Multiplex in a Psoriatic Patient during Treatment with Anti-IL-12/23
}

\author{
Claudio Marasca Matteo Megna Marianna Donnarumma \\ Giuseppina Fontanella Eleonora Cinelli Gabriella Fabbrocini \\ Department of Dermatology, University of Naples Federico II, Naples, Italy
}

\section{Established Facts}

- Steatocystoma multiplex (SM) is an autosomal dominant disorder, marked by multiple asymptomatic cutaneous cysts located on the axillae, groin, trunk, and limbs.

- SM has been associated to a mutation of the keratin 17 gene (KRT17) on chromosome 17q21.21.

- Ustekinumab is an interleukin (IL)-12/IL-23 inhibitor, used in the treatment of psoriasis.

\section{Novel Insights}

- This is the first case described in the literature of SM during ustekinumab treatment.

- Ustekinumab reduces the pro-inflammatory effector cytokines interferon (IFN)- $\gamma$ and interleukin (IL)-17/IL-22, thus reducing upregulation of keratin 17 (K17) in skin tissue.

- Our hypothesis is that ustekinumab promoted SM by reducing the expression of IFN- $\gamma$ and IL-17/IL22 and consequently acting on the K17 pathway.

\section{Keywords}

Steatocystoma · Psoriasis · Ustekinumab · Biologics

\section{Abstract}

Steatocystoma multiplex (SM) is an autosomal dominant disorder developing in adolescence or early adult age. The occurrence of multiple asymptomatic cutaneous cysts on the axillae, groin, trunk, and limbs characterizes the disease. SM is associated with a missense mutation in the keratin 17 gene
(KRT17), a gene encoding for a type I intermediate filament (keratin 17 [K17]), mainly expressed in the epithelial appendages (hair follicles and sebaceous glands). Here, we report a case of appearance of multiple steatocystomas in a psoriatic patient during ustekinumab treatment, an interleukin (IL)12/IL-23 inhibitor. Our hypothesis is that ustekinumab could have unmasked a potential genetic predisposition to SM by reducing the expression of interferon- $\gamma$ and IL-17/IL-22 and consequently acting on the K17 pathway.

(c) 2020 S. Karger AG, Basel karger@karger.com www.karger.com/sad

(C) 2020 S. Karger AG, Basel

Karger ${ }^{\prime}=$
Claudio Marasca

Department of Clinical Medicine and Surgery

Section of Dermatology and Venereology, University of Naples Federico II

Via Pansini 5, IT-80138 Naples (Italy)

claudio.marasca@gmail.com 


\section{Introduction}

Steatocystoma multiplex (SM), described for the first time by Jamieson in 1873, is an autosomal dominant disorder developing in adolescence or early adult age, marked by the occurrence of multiple asymptomatic cutaneous cysts in areas such as the axillae, groin, trunk, and limbs [1].

$\mathrm{SM}$ is associated with a missense mutation in the keratin 17 gene (KRT17), a gene encoding for a type I intermediate filament (keratin 17 [K17]). K17 is mainly expressed in the epithelial appendages (hair follicles and sebaceous glands) [2].

Treatment options include oral isotretinoin, surgical excision, and physical therapies such a $\mathrm{CO}_{2}$ laser and cryotherapy [3]. We report a case of SM in a patient suffering from psoriasis during treatment with ustekinum$\mathrm{ab}$, an interleukin (IL)-12/IL-23 inhibitor.

\section{Case Report}

In February 2019, a 24-year-old Pakistani woman, with a 10-year and family history for psoriasis, attended our outpatient clinic of Dermatology, Department of Clinical Medicine and Surgery, Federico II of Naples, for occurrence of psoriatic lesions involving about $50 \%$ of the body surface along with the palms and genital area, with a total PASI (Psoriasis Area and Severity Index) score of 16.

The patient was previously treated with systemic therapies (methotrexate), topical agents (corticosteroids, emollients and keratolytic products), and phototherapy (narrow-band ultraviolet B) with inadequate response and relapses after therapy suspension.

Moreover, the disease also had a negative impact on the patient's quality of life (QoL), as testified by the Dermatology Life Quality
Index (DLQI) score of 12 points, mainly because of the involvement of special locations (e.g., genital area and palms).

According to the severity of the disease and its impact on the patient's QoL, and because of the inadequate response to even previous systemic therapies, we decided to begin ustekinumab at labeled dosage. The patient received a $45-\mathrm{mg}$ dose subcutaneously at baseline, after 4 weeks, and every 12 weeks thereafter (patient's bodyweight $=56 \mathrm{~kg}$ ).

After 8 weeks of treatment, the patient showed a clear improvement of the skin lesions with a decrease in the PASI score to 6 and an improvement in QoL evaluated through a DLQI score decrease of 6 points (DLQI at week $8=6$ ).

Unfortunately, after 16 weeks of treatment, the patient developed unexpected lesions in the axillary regions (Fig. 1). The lesions consisted in painless multiple, elastic, firm subcutaneous nodules on both axillae. The patient denied a personal and familiar history of similar lesions.

An excisional biopsy of a nodule was performed. Histopathology revealed an epithelial cyst delimited by squamous epithelium and contiguous sebaceous glands. Clinical and histological findings were compatible with SM. Ustekinumab was discontinued, and a topical therapy was provided for psoriasis. After 4 weeks of ustekinumab suspension, disappearance of SM lesions was observed.

\section{Discussion}

Psoriasis is a common chronic skin disease characterized by an abnormal $\mathrm{T}$-cell reaction resulting in inflammation, hyperproliferation, and aberrant differentiation of keratinocytes. The exact pathogenic mechanism is unclear, but studies suggest that keratinocytes, in susceptible individuals, may secrete antimicrobial peptides with activation of plasmacytoid dendritic cells in the dermis following dif-
Fig. 1 Multiple, elastic, firm subcutaneous nodules located at both axillae after 16 weeks of treatment with ustekinumab.

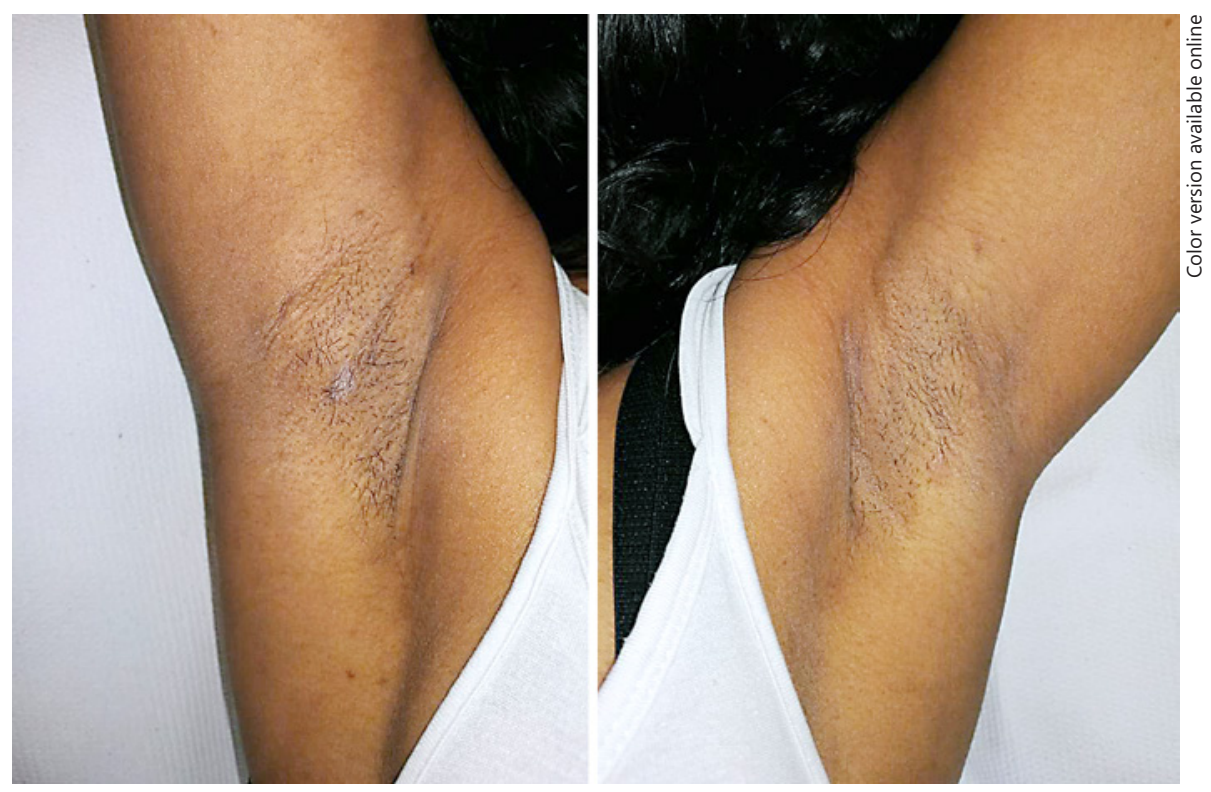

Marasca/Megna/Donnarumma/ Fontanella/Cinelli/Fabbrocini 
ferent stressful events such as microbial infection, trauma, drug usage, or smoking [4].

Subsequently, dendritic cells release IL-12 and 23, which activate 2 types of T cells: T helper 1 and T helper 17 [5]. Involved $\mathrm{T}$ cells begin to produce psoriatic cytokines, including IL-17, interferon (IFN)- $\gamma$, tumor necrosis factor- $\alpha$, and IL-22 [5]. The inflammatory factors in turn promote the expression of K17 in psoriatic epidermis. This autoimmune positive feedback loop confirms that K17 is involved in the development and relapse of psoriasis [6]. K17 is a multifunctional cytoskeletal protein that regulates a myriad of cellular processes, including cell proliferation and growth, skin inflammation, and the differentiation of skin appendages [7, 8]. Indeed, K17 overexpression is observed in inflammatory skin diseases such as psoriasis [9]. Recent findings indicated that K17 could shuttle in and out of the nucleus owing to the existence of a nuclear localization signal and a nuclear export signal. This new idea of K17 as a nuclear intermediate filament raises the possibility that keratins regulate additional cellular processes [2].

Ustekinumab is an IL-12/IL-23 inhibitor: it can act in psoriasis, reducing the pro-inflammatory actions of the effector cytokines IFN- $\gamma$ and IL-17/IL-22 [10]. Consequent$l y$, this cytokine cascade arrest can reduce the upregulation of $\mathrm{K} 17$ in skin tissue.

$\mathrm{K} 17$ expression is involved also in other cutaneous diseases such as SM. In fact, SM results from a mutation of KRT17 on chromosome 17q21.21, and, to date, 17 mutations of KRT17 have been reported and well documented [11]. Therefore, we hypothesized that ustekinumab could have unmasked SM by reducing the expression of IFN- $\gamma$ and IL-17/IL-22, acting on the K17 pathway.

In fact, in this case, the expression of K17 could be influenced by 2 mechanisms: on one hand, psoriatic background contributes to higher K17 expression, but, on the other hand, a possible individual genetic predisposition could lead to an altered K17. When the patient does not assume biologic therapy, the psoriatic milieu prevails, as multiple and different cytokines promote psoriasis. When on ustekinumab therapy, the reduction of IFN- $\gamma$, IL-17/ IL-22, and, subsequently, K17 could have unmasked the genetically altered expression of K17.

This is the first case described in the literature of appearance of multiple steatocystomas in a psoriatic patient during ustekinumab treatment. Further cases and molecular studies are necessary to confirm this pathogenic hypothesis and to better understand genetic predisposing factors and backgrounds.

\section{Statement of Ethics}

The patient gave written informed consent to publish the case.

\section{Conflict of Interest Statement}

The authors have no conflicts of interest to declare.

\section{Funding Sources}

The authors did not receive any funding.

\section{Author Contributions}

Dr. Claudio Marasca contributed to case data acquisition and concepts, and manuscript writing. Dr. Matteo Megna contributed to case data acquisition and concepts. Dr. Marianna Donnarumma wrote and edited the manuscript. Dr. Giuseppina Fontanella edited and reviewed the manuscript. Dr. Eleonora Cinelli edited and reviewed the manuscript. Prof. Gabriella Fabbrocini contributed to case data acquisition and concepts, and manuscript review.

\section{References}

1 Kamra HT, Gadgil PA, Ovhal AG, Narkhede RR. Steatocystoma multiplex: a rare genetic disorder: a case report and review of the literature. J Clin Diagn Res. 2013;7(1):166-8.

2 Yang L, Zhang S, Wang G. Keratin 17 in disease pathogenesis: from cancer to dermatoses. J Pathol. 2019 Feb;247(2):158-65.

3 Lin KP, Chang ME, Ho WT. Treatment of steatocystoma multiplex on axillae using keyhole approach technique. J Cosmet Laser Ther. 2019;21(4):1.

4 Guttman-Yassky E, Nograles KE, Krueger JG. Contrasting pathogenesis of atopic dermatitis and psoriasis: part I: clinical and pathologic concepts. J Allergy Clin Immunol. 2011;127(5): $1110-8$.
5 Gilliet M, Lande R. Antimicrobial peptides and self-DNA in autoimmune skin inflammation. Curr Opin Immunol. 2008 Aug; 20(4):401-7.

6 Zhang W, Dang E, Shi X, Jin L, Feng Z, Hu L, et al. The pro-inflammatory cytokine IL-22 up-regulates keratin 17 expression in keratinocytes via STAT3 and ERK1/2. PLoS One. 2012;7(7):e40797.

7 DePianto D, Kerns ML, Dlugosz AA, Coulombe PA. Keratin 17 promotes epithelial proliferation and tumor growth by polarizing the immune response in skin. Nat Genet. 2010 Oct;42(10):910-4.
8 Tong X, Coulombe PA. Keratin 17 modulates hair follicle cycling in a TNFalpha-dependent fashion. Genes Dev. 2006 May;20(10):135364.

9 Jin L, Wang G. Keratin 17: a critical player in the pathogenesis of psoriasis. Med Res Rev. 2014 Mar;34(2):438-54.

10 Savage LJ, Wittmann M, McGonagle D, Helliwell PS. Ustekinumab in the treatment of psoriasis and psoriatic arthritis. Rheumatol Ther. 2015 Jun;2(1):1-16.

11 Zhang B, Sun L, Fu X, Yu G, Liu H, Zhang F. Mutation analysis of the KRT17 gene in steatocystoma multiplex and a brief literature review. Clin Exp Dermatol. 2020;45(1):132-4.
Steatocystoma Multiplex in a Psoriasis Patient during Ustekinumab
Skin Appendage Disord 2020;6:309-311 DOI: $10.1159 / 000507657$ 